\title{
ON PRIME DIVISORS OF LARGE POWERS OF ELEMENTS IN NOETHER LATTICES
}

\author{
by LINDA BECERRA
}

(Received 20 August, 1987)

1. Introduction. In [4], R. P. Dilworth introduced the concept of a Noether lattice as an abstraction of the lattice of ideals of a Noetherian ring and he showed that many important properties of Noetherian rings, such as the Noether decomposition theorems, also hold for Noether lattices. It was later shown, in [1], that every Noether lattice is not the lattice of ideals of any Noetherian ring, yet many studies have successfully been undertaken to relate other concepts between Noetherian rings and Noether lattices as had been begun by Dilworth. (See [3], [5], and [6].) In this paper we undertake such a study and show that some results of M. Brodmann in [2] and L. Ratliff in [7] concerning prime divisors of large powers of a fixed element of a commutative Noetherian ring may be generalized and extended to the setting of a Noether lattice. It is shown (Theorem 2.8) that if $A$ is an element of a Noether lattice then all large powers of $A$ have the same prime divisors and (Corollary 3.8) included among this fixed set of primes are those primes that are prime divisors of the integral closure of $A^{k}$ for some $k \geqq 1$. We note that the ring proof of this latter result does not generalize directly since it uses the notion of transcendence degree which to our knowledge has no analogue in multiplicative lattices.

Throughout the remainder of this paper, unless otherwise stated, $\mathscr{L}$ will denote a Noether lattice [4], and any prime element of $\mathscr{L}$ will be assumed to be distinct from $I$, the greatest element of $\mathscr{L}$. We note that a prime $P$ is a prime divisor of $A$ in $\mathscr{L}$ if and only if there is a $B$ in $\mathscr{L}$ such that $A: B=P$. Furthermore, if $P$ is a prime divisor of $A$ then $B$ can be taken to be principal. We will make use of this fact many times throughout this paper.

2. All large powers of an element have the same prime divisors. Principal nonzero divisors will play an important role in our work in this paper and we begin with a result on prime divisors of such elements.

Lemma 2.1. Let $P$ be a prime element of $\mathscr{L}$. If $P$ is a prime divisor of a principal nonzero divisor of $\mathscr{L}$, then $P$ is a prime divisor of each principal nonzero divisor $B$ such that $B \leqq P$.

Proof. Suppose $A$ is a principal nonzero divisor and $P$ is a prime divisor of $A$. Then we may choose a $C$ in $\mathscr{L}$ such that $A: C=P$. Consequently, $C P \leqq A$. Now let $B$ be a principal nonzero divisor such that $B \leqq P$. Then $C B \leqq C P \leqq A$, and so $C B=C B \wedge A=$ $(C B: A) A$. Therefore, since $A$ and $B$ are principal nonzero divisors, it follows that $B:(C B: A)=A: C=P$, and this concludes the proof.

In many of the results that we are to show, we make use of E. W. Johnson's $A$-transform of $\mathscr{L}$, which is denoted by $\mathscr{R}(\mathscr{L}, A)$. It consists of all formal sums $\sum_{i=-\infty}^{\infty} B_{i}$ of

Glasgow Math. J. 30 (1988) 359-367. 
elements $B_{i}$ of $\mathscr{L}$ for which the relations $A^{i} \geqq B_{i} \geqq B_{i+1} \geqq A B_{i}$ hold for all integers $i$. In [5], it is shown that if $\mathscr{L}$ is a Noether lattice and $A \in \mathscr{L}$ then $\mathscr{R}(\mathscr{L}, A)$ is a Noether lattice, where, for elements $B, C \in \mathscr{R}(\mathscr{L}, A), B \leqq C$ if and only if $B_{i} \leqq C_{i}$ for all $i$, and

$$
B \vee C=\sum\left(B_{i} \vee C_{i}\right), \quad B \wedge C=\sum\left(B_{i} \wedge C_{i}\right), \quad B \cdot C=\sum_{i}\left(\bigvee_{r+s=i} B_{r} C_{s}\right) .
$$

Furthermore, if $C \leqq A^{r}$ then $C^{[r]}$ is defined to be the least element $D$ of $\mathscr{R}(\mathscr{L}, A)$ such that $C \leqq D_{r}$, and it is shown that $C^{[r]}=\sum_{i} C A^{i-r}$. Other properties of $\mathscr{R}(\mathscr{L}, A)$ proven in [5] that we will need are: (1) $B C^{[r]}=\sum_{i} B_{i-r} C$, (2) $B: C^{[r]}=\sum_{i}\left[\left(B_{i+r}: C\right) \wedge A^{i}\right]$, (3) if $C$ is a principal element of $\mathscr{L}$ such that $C \leqq A^{r}$ then $C^{r l}$ is a principal element of $\mathscr{R}(\mathscr{L}, A)$, and (4) each $B \in \mathscr{R}(\mathscr{L}, A)$ is the join of finitely many principal elements of the form $C^{[r]}$, where $C \leqq A^{r}$ is principal in $\mathscr{L}$.

In the following lemma, information is given that relates prime and primary elements of $\mathscr{R}(\mathscr{L}, A)$ to prime and primary elements of $\mathscr{L}$.

Lemma 2.2. Let $A \in \mathscr{L}$. Then:

(1) if $\sum Q_{i}, \sum P_{i} \in \mathscr{R}(\mathscr{L}, A)$ such that $\sum Q_{i}$ is $\sum P_{i}$-primary then $Q_{0}$ is $P_{0}$-primary in $\mathscr{L}$;

(2) if $P$ is a prime element of $\mathscr{L}$ and if $\Sigma B_{i} \in \mathscr{R}(\mathscr{L}, A)$ such that $P$ is a prime divisor of $B_{0}$ then there exists a prime element $\sum P_{i} \in \mathscr{R}(\mathscr{L}, A)$ such that $\Sigma P_{i}$ is a prime divisor of $\sum B_{i}$ and $P_{0}=P$.

Proof. The proof of (1) is straightforward and we omit the details.

(2) is an immediate consequence of (1) and the fact that meets in $\mathscr{R}(\mathscr{L}, A)$ are defined componentwise. For it follows that a primary decomposition of an element $\sum B_{i}$ in $\mathscr{R}(\mathscr{L}, A)$ produces a primary decomposition of $B_{0}$ in $\mathscr{L}$.

We now present our first main result. It deals with the cardinality of the set of primes that are prime divisors of some power of a fixed element of $\mathscr{L}$.

Lemma 2.3. Let $A \in \mathscr{L}, A \neq I$. Then the following two sets are equal:

$$
S_{1}=\left\{P \in \mathscr{L} \mid P \text { is a prime divisor of } A^{n} \text { for some positive integer } n\right\}
$$

and

$$
S_{2}=\left\{P \in \mathscr{L} \mid P=\left(\sum P_{i}\right)_{0} \text { for some prime divisor } \sum P_{i} \text { of } I^{[-1]} \text { in } \mathscr{R}(\mathscr{L}, A)\right\} .
$$

Consequently, $S_{1}$ has finite cardinality.

Proof. We observe that for each $n \geqq 1, I^{[-n]}$ is a principal nonzero divisor of $\mathscr{R}(\mathscr{L}, A)$.

We first consider set $S_{1}$. Let $n \geqq 1$ and $P \in \mathscr{L}$ such that $P$ is a prime divisor of $A^{n}$. Then in $\mathscr{R}(\mathscr{L}, A)$, since the 0 th component of $I^{[-n]}$ is $A^{n}$, there exists an element $\sum P_{i}$ which is a prime divisor of $I^{[-n]}$ and has $P_{0}=P$ by Lemma 2.2. Consequently $\sum P_{i} \geqq I^{[-n]}=\left(I^{[-1]}\right)^{n}$, and this implies $\sum P_{i} \geqq I^{[-1]}$ since $\sum P_{i}$ is prime. Hence $\sum P_{i}$ is a prime divisor of $I^{[-1]}$ by Lemma 2.1, and so $S_{1} \subseteq S_{2}$. 
Now let $\sum P_{i} \in \mathscr{R}(\mathscr{L}, A)$ such that $\sum P_{i}$ is a prime divisor of $I^{[-1]}$. Then $\sum P_{i}=$ $I^{[-1]}: B^{[r]}$ for some element $B^{[r]}$ of $\mathscr{R}(\mathscr{L}, A)$. Consequently $P_{0}=A^{r+1}: B$, and so $P_{0}$ is a prime divisor of $A^{r+1}$. We observe that if $r+1 \neq 1$ then $P_{0}=I$, which would be a contradiction. Hence $S_{2} \subseteq S_{1}$, and this completes the proof.

The next result deals with prime divisors of zero. Then, in the corollary that follows, we show that, from this result, information can be derived relating prime divisors of consecutive powers of an element.

TheOREM 2.4. Let $B, C, P \in \mathscr{L}$ such that $C \leqq B$ and $P$ is prime. If there exists a principal element $X \leqq B$ such that $P=0: X$, then either there exists a $Y \in \mathscr{L}, 0<Y \leqq C$, such that $P=0: Y$, or there exists an $S \in \mathscr{L}$ such that $P=C: S$.

Proof. Let us suppose that $P=0: X$, where $X$ is principal and $X \leqq B$. If $X \leqq C$ or if $C=0$ then the result clearly holds.

Suppose then that $X \not C$, so $C<B$, and that $C \neq 0$.

We will now construct a chain of elements $A_{i}$ of $\mathscr{L}$ such that $A_{i}: A_{i+1}$ is prime. Let $A_{0}=0$ and then define inductively $A_{i}$ such that $A_{i-1}: A_{i}$ is a maximal element of the set $\left\{A_{i-1}: W \mid A_{i-1}<W \leqq C\right\}$. We can thus obtain a finite chain of elements $A_{i}$ such that $A_{0}=0$ and $A_{n}=C$. This chain can be extended to include $B$. Using the same construction process as before, we can obtain a finite chain of elements $A_{n+i}, 1 \leqq i \leqq m$, where $A_{n+i-1}: A_{n+i}$ is a maximal element of the set $\left\{A_{n+i-1}: W \mid A_{n+i-1}<W \leqq B\right\}$ and $A_{n+m}=B$.

We now show that $P=A_{j}: A_{j+1}$ for some $j$. Let $j$ be chosen such that $X \wedge A_{j}=0$ and $X \wedge A_{j+1} \neq 0$. Now by our choice of the $A_{i}$ and the fact that

$$
A_{j}: A_{j+1} \leqq A_{j}:\left[A_{j+1} \wedge\left(X \vee A_{j}\right)\right],
$$

we must have that either

$$
A_{j}: A_{j+1}=A_{j}:\left[A_{j+1} \wedge\left(X \vee A_{j}\right)\right] \text { or } A_{j+1} \wedge\left(X \vee A_{j}\right)=A_{j} .
$$

But $A_{j}=A_{j+1} \wedge\left(X \vee A_{j}\right)$ would imply $X \wedge A_{j+1}=0$, which would be a contradiction. Hence we may conclude that

$$
\begin{aligned}
A_{j}: A_{j+1} & =A_{j}:\left[A_{j+1} \wedge\left(X \vee A_{j}\right)\right] \\
& =A_{j}:\left[\left(A_{j+1} \wedge X\right) \vee A_{j}\right] \\
& =A_{j}:\left(A_{j+1} \wedge X\right) \\
& \geqq A_{j}: X \geqq 0: X=P .
\end{aligned}
$$

To show that $A_{j}: A_{j+1} \leqq P$, let $U \leqq A_{j}: A_{j+1}=\left[A_{j}:\left(A_{j+1} \wedge X\right)\right]$. Then $U\left(A_{j+1} \wedge X\right) \leqq A_{j}$, and so it follows that

$$
U\left(A_{j+1} \wedge X\right)=U\left(A_{j+1}: X\right) X \leqq A_{j} \wedge X=0 .
$$


Consequently either $U \leqq P$ or $\left(A_{j+1}: X\right) \leqq P$. But $A_{j+1}: X \leqq P$ would imply

$$
X \wedge A_{j+1}=X\left(A_{j+1}: X\right) \leqq P X=0,
$$

which would be a contradiction. Therefore $U \leqq P$ and it follows that $A_{j}: A_{j+1}=P$.

We now consider the cases of whether $j \leqq n-1$ or $j \geqq n$.

Suppose $j \leqq n-1$. Then $j+1 \leqq n$ and so $A_{j+1} \leqq A_{n}=C$. Consequently we have that

$$
P=A_{j}: A_{j+1}=A_{j}:\left(A_{j+1} \wedge X\right) \geqq 0:\left(A_{j+1} \wedge X\right) \geqq 0: X=P .
$$

Letting $Y=\left(A_{j+1} \wedge X\right)$, we have that $P=0: Y$, where $0<Y \leqq C$.

Suppose $j \geqq n$. Then $A_{j} \geqq A_{n}=C$ and it follows, the proof being similar to the case above, that $P=C:\left(A_{j+1} \wedge X\right)$. Letting $S=A_{j+1} \wedge X$, we have that $P=C: S$. This completes the proof.

Corollary 2.5. Let $n$ be a positive integer and $A, P \in \mathscr{L}$ such that $P$ is prime. If $P$ is a prime divisor of $A^{n+1}$, then either $P$ is a prime divisor of $A^{n}$ or there exists a $Y \in \mathscr{L}$, $A^{n+1}<! Y \leqq A^{n}$, such that $P=A^{n+1}: Y$.

Proof. This follows by applying Theorem 2.4 to the elements $C=A^{n}$ and $B=I$ in the Noether lattice $\mathscr{L} / A^{n+1}$.

We now present a computational result concerning powers of a fixed element. We will then be ready to relate prime divisors of large powers of a fixed element.

Lemma 2.6. For each $A \in \mathscr{L}$ there exists a positive integer $n$ such that

for all $i \geqq 1$.

$$
\left(A^{n+i+2}: A\right) \wedge A^{n+i}=A^{n+i+1}
$$

Proof. Let $A \in \mathscr{L}$ and consider the element $I^{[-1]}: A^{[1]}$ of $\mathscr{R}(\mathscr{L}, A)$. By Theorem 2.8 of [5], we may choose a positive integer $n$ such that

$$
\left(I^{[-1]}: A^{[1]}\right)_{n+i}=\left(I^{[-1]}: A^{[1]}\right)_{n} A^{i} \text { for all } i \geqq 0 .
$$

Hence, for all $i \geqq 0$, from (1) we obtain that

$$
\left(A^{n+i+2}: A\right) \wedge A^{n+i}=\left[\left(A^{n+2}: A\right) \wedge A^{n}\right] A^{i} .
$$

Consequently, for each $i \geqq 1$, it follows that

$$
\begin{aligned}
A^{n+1+i} & \leqq\left(A^{n+2+i}: A\right) \wedge A^{n+i} \\
& =\left[\left(A^{n+2}: A\right) \wedge A^{n}\right] A^{i} \\
& \leqq\left(A^{n+2}\right) A^{i-1} \\
& =A^{n+1+i} .
\end{aligned}
$$

Lemma 2.7. Let $A \in \mathscr{L}$. For each $n \geqq 1$, define

$\beta(n)=\left\{P \in \mathscr{L} \mid P\right.$ is prime and there exists a $Y, A^{n}<Y \leqq A^{n-1}$, such that $\left.P=A^{n}: Y\right\}$.

Then there exists a positive integer $m$ such that $\beta(m)=\beta(m+i)$ for all $i \geqq 0$. 
Proof. Let $r$ be a positive integer such that $\left(A^{r+i+2}: A\right) \wedge A^{r+i}=A^{r+i+1}$ for all $i \geqq 1$ (Lemma 2.6). Let $j \geqq 1$ and $P \in \beta(r+j+1)$. Then there exists a $Y, A^{r+j+1}<Y \leqq A^{r+j}$, such that $P=A^{r+j+1}: Y$. Hence

$$
\begin{aligned}
P & =A^{r+j+1}: Y \\
& =\left[\left(A^{r+j+2}: A\right) \wedge A^{r+j}\right]: Y \\
& =\left(A^{r+j+2}: A\right): Y \\
& =A^{r+j+2}: A Y
\end{aligned}
$$

and so $\beta(r+j+1) \subseteq \beta(r+j+2)$. Therefore, since $\cup \beta(n)$ is finite (Lemma 2.3), there exists a positive integer $m$ such that $\beta(m)=\beta(m+i)$ for all $i \geqq 0$, and this completes the proof.

ThEOREM 2.8. Let $A \in \mathscr{L}$. For each $n \geqq 1$, let $\alpha(n)$ denote the set of prime divisors of $A^{n}$. Then there exists a positive integer $r$ such that $\alpha(r)=\alpha(r+i)$ for all $i \geqq 0$.

Proof. Let $m$ be an integer such that $\beta(m)=\beta(m+i)$ for all $i \geqq 1$, where $\beta$ is the function defined in Lemma 2.7. Now let $P$ be a prime divisor of $A^{m+1}$. Then, by Corollary 2.5 , either $P \in \alpha(m)$ or $P \in \beta(m+1)$. Hence

$$
\begin{aligned}
\alpha(m+1) & \subseteq \alpha(m) \cup \beta(m+1) \\
& =\alpha(m) \cup \beta(m) \\
& =\alpha(m) .
\end{aligned}
$$

Therefore, since $\bigcup \alpha(n)$ is finite (Lemma 2.3), there exists an $r$ such that $\alpha(r)=\alpha(r+i)$ for all $i \geqq 0$. This completes the proof.

3. A set of primes that are prime divisors of all large powers of a fixed element. In this section we will see that included in the collection of primes that are prime divisors of all large powers of a fixed element of $\mathscr{L}$ are the prime divisors of the integral closure of that element. Basic properties and results about the integral closure operation may be found in [6] and include the following. If $A \in \mathscr{L}$ then the integral closure of $A$, denoted $A_{a}$, is the join of all elements $B$ in $\mathscr{L}$ for which there exists a positive integer $n$ such that $(A \vee B)^{n+1}=A(A \vee B)^{n}$. It is shown that, in the lattice of ideals of a Noetherian ring, this concept coincides with the usual ring notion of the integral closure of an ideal. Also, if $A$, $B \in \mathscr{L}$ then $A B \leqq A_{a} B_{a} \leqq(A B)_{a}$, and if $C \leqq A^{n}$ then the integral closure of the element $C^{[n]}$ of $\mathscr{R}(\mathscr{L}, A)$ is given by $\left(C^{[n]}\right)_{a}=\sum_{i}\left[\left(C A^{i-n}\right)_{a} \wedge A^{i}\right]$.

Our first result in this section is a type of cancellation law that involves the integral closure operation and this is followed with a result involving a lattice transform of $\mathscr{L}$ whose proof depends on this cancellation law.

Lemma 3.1. Let $A, B, C \in \mathscr{L}$. If there exists a principal element $C_{1} \leqq C_{a}$ with $0: C_{1}=0$ then $\left(A C^{n}\right)_{a} \leqq\left(B C^{n}\right)_{a}$ for some $n \geqq 1$ implies $A_{a} \leqq B_{a}$.

Proof. Suppose $C_{1}$ is a principal nonzero divisor and $C_{1} \leqq C_{a}$. Then $\left(C_{1}\right)^{m}$ is a principal nonzero divisor for each $m \geqq 1$. Also, $C_{1} \leqq C_{a}$ implies $\left(C_{1}\right)^{m} \leqq\left(C_{a}\right)^{m} \leqq\left(C^{m}\right)_{a}$ for 
each $m \geqq 1$. Hence if $\left(A C^{n}\right)_{a} \leqq\left(B C^{n}\right)_{a}$ then $\left(A\left(C^{n}\right)_{a}\right)_{a} \leqq\left(B\left(C^{n}\right)_{a}\right)_{a}$, and consequently it follows that $A_{a} \leqq B_{a}$ by Theorem 1 of [3].

LeMmA 3.2. Let $A \in \mathscr{L}$ such that there exists a principal nonzero divisor $B \leqq A_{a}$. Let $\Sigma P_{i}$ be a proper element of $\mathscr{R}(\mathscr{L}, A)$ such that $\sum P_{i}=\left(I^{\mid-k]}\right)_{a}: X^{[t]}$ for some positive integer $k$ and element $X^{[t]}$. Then:

(1) $P_{1}<A$;

(2) if $\sum P_{i}$ is also prime then $\sum P_{i}=\sum P_{i}:\left(A^{[1]}\right)^{i}$ for all $j \geqq 1$.

Proof. Using Theorem 4 of [6], we have that

$$
\begin{aligned}
\sum P_{i} & =\left(I^{[-k]}\right)_{a}: X^{[t]} \\
& =\sum\left[\left(A^{i+k+t}\right)_{a} \wedge A^{i+t}\right]: X \wedge A^{i} .
\end{aligned}
$$

Suppose $P_{1}=A$. Then, using (2) to evaluate the first component of $\sum P_{i}$, we would have that $\left[\left(A^{1+k+t}\right)_{a} \wedge A^{1+t}\right]: X \wedge A=A$. It would follow that $A X \leqq\left(A^{1+k+t}\right)_{a}$. But this implies $(A X)_{a} \leqq\left(A^{1+k+t}\right)_{a}$, and consequently it would follow that $X \leqq\left(A^{k+t}\right)_{a}$ by Lemma 3.1, which would be a contradiction to our choice of $\sum P_{i}$. Hence $P_{1}<A$.

For the proof of (2), let us assume that $\sum P_{i}$ is prime. Since $P_{1}<A$, it follows that $\left(A^{[1]}\right) \sum P_{i}$. Consequently $\left(A^{[1]}\right)^{j} \$ \sum P_{i}$ for all $j$ since $\sum P_{i}$ is prime. Therefore $\sum P_{i}=\sum P_{i}:\left(A^{[1]}\right)^{j}$ for all $j \geqq 1$ since, for any $C, D \in \mathscr{L}, C: D=C$ if and only if, for every prime divisor $R$ of $C, D \nsubseteq R$. This completes the proof.

In the next two results, first in the local and then in the nonlocal case, we deal with primes of the integral closure of large powers of an element. From these we will derive information about primes of large powers of an element.

Lemma 3.3. Let $\mathscr{L}$ be a local Noether lattice with maximal element $P$ and $A \in \mathscr{L}$ such that there exists a principal nonzero divisor $B$ with $B \leqq A_{a}$. If $P$ is a prime divisor of $\left(A^{n}\right)_{a}$ for some positive integer $n$, then $P$ is a prime divisor of $\left(A^{m}\right)_{a}$ for all $m \geqq n$.

Proof. Let $n \geqq 1$ and suppose $P$ is a prime divisor of $\left(A^{n}\right)_{a}$. Then we may choose an $X \in \mathscr{L}$ such that $\left(A^{n}\right)_{a}: X=P$.

Now let $i \geqq 1$. We note that

$$
\left[X\left(A^{i}\right)_{a}\right]\left[\left(A^{n}\right)_{a}: X\right] \leqq\left(A^{i}\right)_{a}\left(A^{n}\right)_{a} \leqq\left(A^{n+i}\right)_{a} .
$$

Consequently,

$$
\left(A^{n+i}\right)_{a}:\left[X\left(A^{i}\right)_{a}\right] \geqq\left(A^{n}\right)_{a}: X=P .
$$

Therefore, since $\mathscr{L}$ is local, it follows from (3) that either $\left(A^{n+i}\right)_{a}:\left[X\left(A^{i}\right)_{a}\right]=P$ or $X\left(A^{i}\right)_{a} \leqq\left(A^{n+i}\right)_{a}$. But $X\left(A^{i}\right)_{a} \leqq\left(A^{n+i}\right)_{a}$ implies $\left(X A^{i}\right)_{a} \leqq\left(A^{n+i}\right)_{a}$, and it would follow that $X \leqq\left(A^{n}\right)_{a}$ by Lemma 3.1, which would be a contradiction. Hence $P=\left(A^{n+i}\right)_{a}:\left[X\left(A^{i}\right)_{a}\right]$ for each $i \geqq 1$, and this concludes the proof. 
THEOREM 3.4. Let $A \in \mathscr{L}$ such that there exists a principal nonzero divisor $B \leqq A_{a}$. If $P$ is a prime divisor of $\left(A^{n}\right)_{a}$ for some positive integer $n$, then there exists a positive integer $m$ such that $P$ is a prime divisor of $\left(A^{m+i}\right)_{a}$ for all $i \geqq 0$.

Proof. Let us suppose $P$ is a prime divisor of $\left(A^{n}\right)_{a}$. We will assume $P$ is not the unique maximal prime of $\mathscr{L}$ since the result would then follow from Lemma 3.3.

Let us now consider the element $\left(I^{[-n]}\right)_{a}$ of $\mathscr{R}(\mathscr{L}, A)$. By Theorem 4 of [6], $\left(I^{[-n]}\right)_{a}=\sum\left[\left(A^{i+n}\right)_{a} \wedge A^{i}\right]$. Hence $\left(A^{n}\right)_{a}$ is the 0 th component of $\left(I^{[-n]}\right)_{a}$. Consequently we may choose an element $\sum P_{i}$ in $\mathscr{R}(\mathscr{L}, A)$ such that $\sum P_{i}$ is a prime divisor of $\left(I^{[-n]}\right)_{a}$ and $P_{0}=P$ by Lemma 2.2. It follows that $\sum P_{i}=\left(I^{[-n]}\right)_{a}: H^{[r]}$ for some element $H^{[r]}$ of $\mathscr{R}(\mathscr{L}, A)$. Thus, since $P$ is a proper element of $\mathscr{L}$, it follows from Lemma 3.2 that $\sum P_{i}=\sum P_{i}:\left(A^{[1]}\right)^{j}$ for all $j \geqq 1$. This may be rewritten as

$$
\begin{aligned}
\sum P_{i} & =\sum P_{i}:\left(A^{[1]}\right)^{j} \\
& =\left[\left(I^{[-n]}\right)_{a}: H^{[r]}\right]:\left(A^{[1]}\right)^{j} \\
& =\left(I^{[-n]}\right)_{a}:\left[H^{[r]}\left(A^{j}\right)^{[j]}\right] \\
& =\left(I^{[-n]}\right)_{a}:\left(H A^{j}\right)^{[r+j]} \\
& =\sum\left(\left[\left(A^{i+n+r+j}\right)_{a} \wedge A^{i+r+j}\right]: H A^{j}\right) \wedge A^{i} .
\end{aligned}
$$

Therefore, using (4) and the fact that the 0th component of $\sum P_{i}$ is $P$, we have that, for each $j \geqq 1$,

This concludes the proof.

$$
\begin{aligned}
P & =\left[\left(A^{n+r+j}\right)_{a} \wedge A^{r+j}\right]: H A^{j} \\
& =\left(A^{n+r+j}\right)_{a}: H A^{j} .
\end{aligned}
$$

In our last four results, we show that under certain conditions prime divisors of the integral closure of an element are consequently prime divisors of large powers of that element.

Lemma 3.5. Let $\mathscr{L}$ be a local Noether lattice with maximal element $P$. If $P$ is a prime divisor of $A_{a}$, where $A$ is a principal nonzero divisor of $\mathscr{L}$, then there exists a $C \in \mathscr{L}$ and a positive integer $k$ such that $A^{k+m}: C A^{m}=P$ for each $m \geqq 0$.

Proof. Let us assume the hypothesis of the lemma. Then we may choose an $X \in \mathscr{L}$ such that $A_{a}: X=P$. This implies $X P \leqq A_{a}$ and so, see [6, Theorem 2 and Theorem 3], there exists a positive integer $j$ such that $(X P)^{j+i} \leqq A^{i}$ for all $i \geqq 0$. It follows that $P^{j+i} \leqq A^{i}: X^{j+i}$, and this implies $P \leqq \sqrt{A^{i}: X^{j+i}}$. Hence, since $\mathscr{L}$ is local, for each $i$ we must have that either

$$
\sqrt{A^{i}: X^{j+i}}=P \text { or } \sqrt{A^{i}: X^{j+i}}=I .
$$

Let us suppose $\sqrt{A^{i}: X^{j+i}}=I$ for each $i \geqq 0$. It would follow that $X^{j+i} \leqq A^{i}$ for each $i$. Consequently we would have that $X \leqq A_{a}$, which would be a contradiction to our choice of $P$. 
Thus there exists an integer $k$ such that $P=\sqrt{A^{k}: X^{j+k}}$. It follows that $P$ is a prime divisor of $A^{k}$ and so there exists a $C \in \mathscr{L}$ such that $A^{k}: C=P$. Therefore, for each $m \geqq 0$, since $A^{m}$ is a principal nonzero divisor, we have that

$$
\begin{aligned}
A^{k+m}:\left(C A^{m}\right) & =\left(A^{k} A^{m}\right):\left(C A^{m}\right) \\
& =\left[\left(A^{k} A^{m}\right): A^{m}\right]: C \\
& =A^{k}: C=P .
\end{aligned}
$$

This completes the proof.

Corollary 3.6. Let $A, P \in \mathscr{L}$ such that $A$ is a principal nonzero divisor. If $P$ is a prime divisor of $A_{a}$ then there exists a positive integer $k$ such that $P$ is a prime divisor of $A^{k+n}$ for each $n \geqq 0$.

Proof. Since $P$ is a prime divisor of an element $B$ of $\mathscr{L}$ if and only if $[P]$ is a prime divisor of $[B]$ in $\mathscr{L}_{P}$, this result follows by applying Lemma 3.5 to $[P]$ and the integral closure of the principal nonzero divisor $[A]$ in $\mathscr{L}_{P}$, which equals $\left[A_{a}\right]$.

THEOREM 3.7. Let $A, P \in \mathscr{L}$ such that there exists a principal nonzero divisor $B \leqq A_{a}$. If $P$ is a prime divisor of $\left(A^{k}\right)_{a}$ for some positive integer $k$ then there exists $a C \in \mathscr{L}$ and a positive integer e such that $A^{e+m}: C A^{m}=P$ for all $m \geqq 0$.

Proof. Let us suppose that $P$ is a prime divisor of $\left(A^{k}\right)_{a}$. Then, since $\left(A^{k}\right)_{a}$ is the 0th component of $\left(I^{[-k]}\right)_{a}$ of $\mathscr{R}(\mathscr{L}, A)$, we may choose an element $\sum P_{i}$ in $\mathscr{R}(\mathscr{L}, A)$ such that $\sum P_{i}$ is a prime divisor of $\left(I^{[-k]}\right)_{a}$ and $P_{0}=P$. Consequently since $P$ is a proper element of $\mathscr{L}$, it follows from Lemma 3.2 that for each $m \geqq 1$ we have

$$
\sum P_{i}=\sum P_{i}:\left(A^{m}\right)^{[m]} \text {. }
$$

Also, since $\sum P_{i}$ is a prime divisor of $\left(I^{[-k]}\right)_{a}$ and $I^{[-k]}$ is a principal nonzero divisor of $\mathscr{R}(\mathscr{L}, A)$, it follows from Corollary 3.6 that there exists a positive integer $s$ such that $\sum P_{i}$ is a prime divisor of $\left(I^{[-k]}\right)^{s}$. Consequently $\sum P_{i}=\left(I^{[-k]}\right)^{s}: C^{[r]}$ for some element $C^{[r]}$ in $\mathscr{R}(\mathscr{L}, A)$, and substituting this for $\sum P_{i}$ into (5) we obtain that, for each $m \geqq 1$,

$$
\begin{aligned}
\sum P_{i} & =\sum P_{i}:\left(A^{m}\right)^{[m]} \\
& =\left(\left(I^{[-k]}\right)^{s}: C^{[r]}\right):\left(A^{m}\right)^{[m]} \\
& =I^{[-k s]}:\left(C A^{m}\right)^{[r+m]} \\
& =\sum\left(A^{i+k s+r+m}: C A^{m}\right) \wedge A^{i} .
\end{aligned}
$$

Therefore, using (6) to determine the 0 th component of $\sum P_{i}$, which is already known to be $P$, we obtain that $P=A^{k s+r+m}: C A^{m}$ for all $m \geqq 1$, and this concludes the proof.

Corollary 3.8. Let $A, P \in \mathscr{L}$ such that there exists a principal nonzero divisor $B \leqq A_{a}$. If $P$ is a prime divisor of $\left(A^{k}\right)_{a}$ for some positive integer $k$ then:

(1) if $D \in \mathscr{L}$ with $D_{a}=A_{a}$ then $P$ is a prime divisor of $D^{n}$ for all large $n$; 
(2) there exists a positive integer $t$ such that if $D \in \mathscr{L}$ with $D_{a}=\left(A^{r}\right)_{a}$ for some $r \geqq t$ then $P$ is a prime divisor of $D^{n}$ for all large $n$.

Proof. Let us suppose that $P$ is a prime divisor of $\left(A^{k}\right)_{a}$.

For the proof of (1), let $D \in \mathscr{L}$ such that $D_{a}=A_{a}$. Then $\left(D^{m}\right)_{a}=\left(A^{m}\right)_{a}$ for all $m \geqq 1$. Hence $P$ is a prime divisor of $\left(D^{k}\right)_{a}$, and consequently $P$ is a prime divisor of $D^{n}$ for all large $n$ by Theorem 3.7.

For the proof of (2), let $t$ be a positive integer such that $P$ is a prime divisor of $\left(A^{t+i}\right)_{a}$ for all $i \geqq 0$ (Theorem 3.4). Now suppose $D \in \mathscr{L}$ such that $D_{a}=\left(A^{r}\right)_{a}$ for some $r \geqq t$. Then $P$ is a prime divisor of $D_{a}$ and so $P$ is a prime divisor of $D^{n}$ for all large $n$ by Theorem 3.7. This completes the proof.

\section{REFERENCES}

1. K. P. Bogart, Structural theorems for regular local Noether lattices, Michigan Math. J. 15 (1968), 167-176. 16-18.

2. M. Brodmann, Asymptotic stability of $\operatorname{Ass}\left(M / I^{n} M\right)$, Proc. Amer. Math. Soc. 74 (1979),

3. R. G. Burton, Integral closure in multiplicative lattices, Algebra Universalis 6 (1976), 399-409.

4. R. P. Dilworth, Abstract commutative ideal theory, Pacific J. Math. 12 (1962), 481-498.

5. E. W. Johnson, A-transforms and Hilbert functions in local lattices, Trans. Amer. Math. Soc. 137 (1969), 125-139.

6. J. P. Lediaev, Asymptotic and integral closure of elements in multiplicative lattices, Michigan Math. J. 16 (1969), 235-243.

7. L. J. Ratliff, Jr, On prime divisors of $I^{n}, n$ large, Michigan Math. J. 23 (1976), 337-352.

UNIVERSITY OF HOUSTON-DOWNTOWN

Houston, TeXas

USA 